\title{
CONFLUENT FLOWS OF A VISCOUS FLUID:
}

\section{A SPECIAL CASE*}

\author{
BY \\ K. GOVINDARAJU \\ University of Maryland-Eastern Shore, Princess Anne
}

1. Introduction. By using the streamlines $\psi(x, y)=$ constant and an arbitrary system of curves $\phi(x, y)=$ constant as a pair of curvilinear coordinates, the Navier-Stokes equations for the steady plane flow of a viscous incompressible fluid of density $\rho$ and coefficient of viscosity $\mu$ may be written as [1]

$$
\begin{gathered}
\left(\frac{\tilde{F}}{\tilde{J}}\right)_{\phi}-\left(\frac{\tilde{E}}{\tilde{J}}\right)_{\psi}=\frac{\omega}{2 k} \frac{\tilde{E}}{\tilde{J}} \quad \text { (vorticity), } \\
\left(\frac{\tilde{G}}{\tilde{J}} \frac{p_{\phi}}{k}-\frac{\tilde{F}}{\tilde{J}} \frac{p_{\psi}}{k}\right)_{\phi}+\left(-\frac{\tilde{F}}{\tilde{J}} \frac{p_{\phi}}{k}+\frac{\tilde{E}}{\tilde{J}} \frac{p_{\psi}}{k}\right)_{\psi}=\mu \frac{\partial(\omega, 1 / k)}{\partial(\phi, \psi)} \quad \text { (Gauss), }
\end{gathered}
$$

where $\omega$ is the vorticity, $k=\frac{1}{2} \rho q^{2}$ is the kinetic energy, $p$ is the pressure and

$$
\begin{array}{ll}
\tilde{E}=\mu^{2} \omega_{\phi}^{2}+h_{\phi}^{2}, & \tilde{F}=\mu^{2} \omega_{\phi} \omega_{\psi}+h_{\phi}\left(h_{\psi}+\omega\right), \\
\tilde{G}=\mu^{2} \omega_{\psi}^{2}+\left(h_{\psi}+\omega\right)^{2}, & \tilde{J}=\psi \omega_{\phi}\left(h_{\psi}+\omega\right)-\mu \omega_{\psi} h_{\phi},
\end{array}
$$

in which $h=k+p$ is the energy.

Once solutions $\omega(\phi, \psi), k(\phi, \psi)$ and $p(\phi, \psi)$ of $(1.1)$ are found, the flow in the physical plane is given by

$$
z=x+i y= \pm \int \frac{e^{i x}}{\sqrt{2 \rho k}}\left|\frac{\tilde{E}}{\widetilde{J}} d \phi+\left(\frac{\tilde{F}}{\tilde{J}}+i\right) d \psi\right|
$$

where

$$
\alpha_{\phi}=\frac{1}{2 k}\left\lfloor-\frac{-\tilde{F} p_{\phi}+\tilde{E} p_{\psi}}{\tilde{J}}+\mu \omega_{\phi}\right\rfloor, \quad \alpha_{\psi}=\frac{1}{2 k}\left[\frac{\tilde{G} p_{\phi}-\tilde{F} p_{\psi}}{\tilde{J}}+\mu \omega_{\psi}\right\rfloor .
$$

Following the designation of Martin [2], we shall call a flow confluent if any two of the following curves coincide in the physical plane:

(a) curves of constant pressure $p$,

(b) streamlines,

(c) curves of constant speed $q$,

(d) curves of constant energy $h$,

* Received January 7, 1980. This research was supported by the National Science Foundation under grant SER76-08595 to the University of Maryland. 
(e) curves of constant direction $\theta$,

(f) curves of constant vorticity $\omega$. Otherwise the flows are called fluent.

Two types of confluent flows $\omega=\omega(\psi)$ and $\omega=\omega(h)$ were discussed in [3]. Calculations show that in these flows the vorticity $\omega$ remains constant along the isovels $q=$ const. It has also been shown that there exist Prandtl-Meyer flows in which the vorticity has a constant value along the isovels. Thus the confluent flow $\omega=\omega(q)$ presents itself for investigation, and this article is devoted to the discussion of the solutions of Eqs. (1.1) with the additional condition $\omega=\omega(q)$ or, equivalently, $k=k(\omega)$.

We distinguish two cases: (i) the streamlines and isobars do not coincide, in which case we make $\phi=p$ in (1.1), and (ii) the streamlines and isobars coincide, $p=p(\psi)$. In the later case we use orthogonal curvilinear coordinates $\phi$ and $\psi$. In either case the requirement $k=k(\omega)$ makes the system over-determined.

Systems of partial differential equations and the existance of their solutions were studied by Riquier [4] and Thomas [5]. Following these methods and using a series of integrability conditions, we obtain solutions in both cases. It will be shown that in the first case the flow will be a Jeffery flow $[6,7]$ and in the second case either the vorticity or the pressure remain constant everywhere in the flow.

I would like to thank Prof. Martin at this time for his valuable suggestions and the National Science Foundation whose grant made this work possible.

2. Streamlines and isobars do not coincide. Using the isobars $p=$ constant in place of the curvilinear coordinates $\phi=$ constant, the system (1.1) becomes

$$
\begin{aligned}
& \left(\frac{\tilde{F}}{\tilde{J}}\right)_{p}-\left(\frac{\tilde{E}}{\tilde{J}}\right)_{\psi}=\frac{\omega}{2 k} \frac{\tilde{E}}{\widetilde{J}} \quad \text { (vorticity), } \\
& \left(\frac{\tilde{G}}{k \widetilde{J}}\right)_{p}-\left(\frac{\tilde{F}}{k \widetilde{J}}\right)_{\psi}=\mu \frac{\partial(\omega, 1 / k)}{\partial(p, \psi)} \quad \text { (Gauss), }
\end{aligned}
$$

where

$$
\begin{array}{ll}
\tilde{E}=\mu^{2} \omega_{p}^{2}+\left(k_{p}+1\right)^{2}, & \tilde{F}=\mu^{2} \omega_{p} \omega_{\psi}+\left(k_{p}+1\right)\left(k_{\psi}+\omega\right), \\
\tilde{G}=\mu^{2} \omega_{\psi}^{2}+\left(k_{\psi}+\omega\right)^{2}, & \tilde{J}=\mu \omega_{p}\left(k_{\psi}+\omega\right)-\mu \omega_{\psi}\left(k_{p}+1\right) .
\end{array}
$$

The assumption $k=k(\omega)$ now implies

$$
k_{p} \omega_{\psi}-k_{\psi} \omega_{p} \equiv 0 .
$$

We shall assume that $\omega_{p} \neq 0$ and $k_{\psi}$ 丰 0 since these cases, $\omega=\omega(\psi)$ and $h=h(\omega)$, have already been discussed in [3]. Thus by (2.2), we have $k_{p} \neq 0$ and $\omega_{\psi} \neq$ 丰 0 . Now, let

$$
\omega_{\psi} / \omega_{p}=k_{\psi} / k_{p}=\lambda(p, \psi)
$$

so that

$$
\begin{array}{ll}
\omega_{\psi}=\lambda \omega_{p}, & \omega_{p \psi}=\lambda_{p} \omega_{p}+\lambda \omega_{p p}, \quad \omega_{\psi \psi}=\lambda_{\psi} \omega_{p}+\lambda \lambda_{p} \omega_{p}+\lambda^{2} \omega_{p p}, \\
k_{\psi}=\lambda k_{p}, & k_{p \psi}=\lambda_{p} k_{p}+\lambda k_{p p}, \quad k_{\psi \psi}=\lambda_{\psi} k_{p}+\lambda \lambda_{p} k_{p}+\lambda^{2} k_{p p} .
\end{array}
$$

We shall now proceed as follows: the vorticity equation together with the Gauss equation will yield $\omega_{p}$ as a function of $\omega$ and $\lambda$. The integrability condition on $\omega_{p}$ and $\omega_{\psi}$ 
given in (2.4) will yield a linear equation for $\lambda_{p}$ and $\lambda_{\psi}$. Replacing the derivatives of $\omega$ in the Gauss equation, we arrive at another linear equation for $\lambda_{p}$ and $\lambda_{\psi}$. The integrability condition $\lambda_{p \psi}=\lambda_{\psi p}$ will yield the condition $\lambda=\lambda(\omega)$. In Lemma 2.1 we show that in this case $\lambda$ must reduce to a constant so that $\omega=\omega(p+\lambda \psi)$ and $k=k(p+\lambda \psi)$.

By means of (2.2), the Gauss equation in (2.1) becomes

$$
\left[\left.\frac{\mu^{2} \omega_{\psi}^{2}+\left(k_{\psi}+\omega\right)^{2}}{k\left(\omega \omega_{p}-\omega_{\psi}\right)}\right|_{p}-\left[\left.\frac{\mu^{2} \omega_{p} \omega_{\psi}+\left(k_{p}+1\right)\left(k_{\psi}+\omega\right)}{k\left(\omega \omega_{p}-\omega_{\psi}\right)}\right|_{\psi}=0\right.\right.
$$

which may be simplified, by using (2.4), to

$$
\begin{aligned}
k \omega \omega_{p}\left(\lambda_{p} \tilde{F}-\lambda_{\psi} \tilde{E}\right)-(\omega-\lambda)^{2}\left(\lambda k_{p}+\omega\right)\left(k \omega_{p}\right)_{p} & \\
& +k \omega_{p}(\omega-\lambda)\left[\omega\left(k_{p}+1\right) \lambda_{p}+\lambda k_{p p}+\omega_{p}\right]=0 .
\end{aligned}
$$

On the other hand, the vorticity equation in (2.1), with the help of $(2.4)$, reduces to

$$
\frac{\omega}{2 k} \frac{\tilde{E}}{\tilde{J}}=\left\lfloor\left.\frac{k_{p}+1}{\omega_{p}}\right|_{p}+\frac{\lambda_{p}\left(k_{p}+1\right)}{\omega_{p}(\omega-\lambda)}+\frac{\lambda_{p} \tilde{E}-\lambda_{\psi} \tilde{E}}{\omega_{p}(\omega-\lambda)^{2}} .\right.
$$

Using (2.5) to eliminate the last term in the above equation and adopting the notation $^{\prime}=d / d \omega$, so that

we may rewrite $(2.6)$ as

$$
k_{p}=k^{\prime} \omega_{p} \quad \text { and } \quad k_{p p}=k^{\prime \prime} \omega_{p}^{2}+k^{\prime} \omega_{p p},
$$

$$
\left[\mu^{2} \omega^{2}+(\omega-\lambda)^{2}\left(k^{\prime 2}-2 k k^{\prime \prime}\right)+\lambda^{2} k^{\prime 2}\right] \omega_{p}^{2}+2\left[(\omega-\lambda)\left(k-\omega k^{\prime}\right)+\omega k^{\prime}\right] \omega_{p}+\omega^{2}=0 .
$$

This is a quadratic for $\omega_{p}$ and is not an identity, since $\omega \neq 0$ by our assumptions. Thus $\omega_{p}$ may be solved as a function $\eta$ of $\omega$ and $\lambda$, so that

$$
\omega_{p}=\eta(\omega, \lambda), \quad \omega_{\psi}=\lambda \cdot \eta(\omega, \lambda) \quad \text { by }(2.4) .
$$

The integrability condition $\omega_{p \psi}=\omega_{\psi p}$ on (2.9) will now yield

$$
\left(\eta+\lambda \eta_{\lambda}\right) \lambda_{p}-\eta_{\lambda} \lambda_{\psi}=0 .
$$

We eliminate the derivatives of $k$ from Eq. (2.5) using (2.7) and the derivatives of $\omega$ using (2.9) to get a second linear equation for $\lambda_{p}$ and $\lambda_{\psi}$ :

$$
\begin{aligned}
& \left\{( \omega - \lambda ) \left[\left(k^{\prime} \eta+1\right) \eta-\left(\omega-\lambda\left(\eta_{\lambda}\right]+\eta\left[\mu^{2} \lambda \eta^{2}+\left(k^{\prime} \eta+1\right)\left(\lambda k^{\prime} \eta+\omega\right]\right\} \lambda_{p}\right.\right.\right. \\
& \quad-\eta\left[\mu^{2} \eta^{2}+\left(k^{\prime} \eta+1\right)^{2}\right] \lambda_{\psi}=\frac{\eta(\omega-\lambda)^{2}}{k \omega}\left[\lambda \eta^{2}\left(k^{\prime 2}-k k^{\prime \prime}\right)+\eta\left(\omega k^{\prime}-k\right)+\omega k \eta_{\omega}\right] .
\end{aligned}
$$

The determinant of the coefficient matrix of $(2.10)$ and (2.11) is given by

$$
-\left[\left(k^{\prime} \eta+1\right) \eta-(\omega-\lambda) \eta_{\lambda}\right]^{2}-\mu^{2} \eta^{4},
$$

which obviously is non-zero. Thus $\lambda_{p}$ and $\lambda_{\psi}$ may be solved as functions of $\omega$ and $\lambda$ :

where

$$
\lambda_{p}=\Delta \cdot \eta_{\lambda}, \quad \lambda_{\psi}=\Delta\left(\eta+\lambda \eta_{\lambda}\right)
$$

$$
\Delta=\frac{\eta(\omega-\lambda)^{2}\left[\lambda \eta^{2}\left(k k^{\prime \prime}-k^{\prime 2}\right)+\eta\left(k-\omega k^{\prime}\right)-\omega k \eta_{\omega}\right]}{k \omega\left[\left(k^{\prime} \eta+1\right) \eta-(\omega-\lambda) \eta_{\lambda}\right]^{2}+\mu^{2} k \omega \eta^{4}} .
$$


We may now apply the integrability condition $\lambda_{p \psi}=\lambda_{\psi p}$ on (2.12) and use (2.9) and (2.12) to eliminate the derivatives of $\omega$ and $\lambda$ to arrive at the equation

$$
\Delta^{2} \eta \eta_{\lambda i}=\Delta_{i} \eta^{2}+2 \Delta^{2} \eta_{\lambda}^{2}+\Delta \eta \eta_{\omega},
$$

which, by rearranging the terms and dividing by $\Delta^{2} \eta^{3}$, may be written as

$$
\left(\frac{1}{\eta}\right)_{i i}=\left(\frac{1}{\Delta \eta}\right)_{\omega} \text {. }
$$

Thus Eq. (2.14) is a necessary condition for the over-determined system (2.1) and (2.2) to be compatible. It involves $\lambda$ and $\omega$ only and thus, if $(2.14)$ is not an identity, $\lambda$ may be solved as a function of $\omega$.

Now, if $\lambda=\lambda(\omega)$, then $\eta=\eta(\omega)$ so that by (2.12) we have

$$
\lambda_{p}=\lambda^{\prime} \omega_{p} \equiv 0,
$$

so that $\lambda=$ constant. We shall state this as a lemma:

Lemma 2.1 If the function $\lambda$ given by (2.3) depends only on the vorticity $\omega$, then $\lambda=$ constant and

$$
\omega=\omega(\tau), \quad k=k(\tau)
$$

where $\tau=p+\lambda \psi$.

We shall now show that under the assumptions already made, $(2.14)$ cannot be an identity.

Since (2.14) involves $1 / \eta$, we shall divide (2.8) by $\omega_{p}^{2}$ and solve for $1 / \omega_{p}$. Thus

$$
\left.\frac{1}{\omega_{p}}=\frac{1}{\eta}=-\mid \frac{k}{\omega}+\lambda\left(\frac{k}{\omega}\right)^{\prime}\right\rfloor \pm \sqrt{ } Q,
$$

where

$$
\begin{array}{ll}
Q=A \lambda^{2}+B \lambda+C, & A=\left(\frac{k}{\omega}\right)^{\prime 2}-2\left(\frac{k^{\prime 2}-k k^{\prime \prime}}{\omega^{2}}\right), \\
B=\left(\frac{k^{2}}{\omega^{2}}\right)^{\prime}+2\left(\frac{k^{\prime 2}-2 k k^{\prime \prime}}{\omega}\right), & C=\frac{k^{2}}{\omega^{2}}-\left(\mu^{2}+k^{\prime 2}-2 k k^{\prime \prime}\right) .
\end{array}
$$

Some useful relations involving these are:

$$
A \omega^{2}+B \omega+C=-\mu^{2}, \quad 2 A \omega+B=-2 k k^{\prime} / \omega^{2}, \quad B^{2}-4 A C=4 \mu^{2} A+\left(4 k^{2} k^{\prime 2} / \omega^{4}\right) .
$$

The first of these relations shows that $A, B$ and $C$ cannot vanish simultaneously for viscous flows.

We note that if $Q \equiv 0$ then $\lambda=\lambda(\omega)$ and by Lemma 2.1 , it reduces to a constant. Thus, assuming that $Q$ 丰 0 , we have

$$
(1 / \eta)_{\lambda \lambda}=\left(4 A C-B^{2}\right) / 4 Q^{3 / 2} .
$$

Further, from (2.13), after eliminating $\eta$ and its derivatives by means of (2.15), we have

$$
1 / \Delta \eta=T / \sqrt{ } Q\left(-2 \lambda \sqrt{ } Q \pm Q_{1}\right)
$$


where

$$
\begin{gathered}
T=\omega^{3}\left(B^{2}-4 A C\right) / 2 k, \quad Q_{1}=A_{1} \lambda^{2}+B_{1} \lambda+C_{1}, \\
A_{1}=2\left(\frac{\omega}{k}\right)^{\prime} A+\frac{\omega}{k} A^{\prime}, \quad B_{1}=2\left(\frac{\omega}{k}\right)^{\prime} B+\frac{\omega}{k} B^{\prime}, \quad C_{1}=2\left(\frac{\omega}{k}\right)^{\prime} C+\frac{\omega}{k} C^{\prime} .
\end{gathered}
$$

We are now in a position to calculate (2.14) with the help of (2.18) and (2.19). Thus, using $\omega$ as a subscript to denote partial differentiation with respect to $\omega$, we have

$$
\begin{aligned}
& 4 \lambda \sqrt{ } Q\left[-T^{\prime} Q+T Q_{\omega}\right] \pm 2 Q \omega\left[\omega T^{\prime} Q_{1}-2 T Q_{1}-\omega T Q_{1, \omega}\right] \mp \omega^{2} T Q_{1} Q_{1, \omega} \\
& =-\frac{k T}{\omega^{3}}\left[-2 \lambda \sqrt{ } Q \pm \omega^{2} Q_{1}\right]^{2}
\end{aligned}
$$

or, by rearranging the terms,

$$
\begin{aligned}
& 4 \lambda \sqrt{ } Q\left|T Q_{\omega}-T^{\prime} Q \mp \frac{k T}{\omega} Q_{1}\right| \\
& = \pm \omega^{2} T Q_{1} Q_{\omega} \mp 2 \omega Q\left[\omega T^{\prime} Q_{1}-2 T Q_{1}-\omega T Q_{1, \omega}\right]-\frac{4 \lambda^{2} k T Q}{\omega^{3}}-\omega k T Q_{1}^{2} .
\end{aligned}
$$

We wish to show that (2.21) is not an identity so that by Lemma $2.1, \lambda$ will reduce to a constant. However, if (2.21) is an identity, since the right side of this equation is fourth-degree in $\lambda$, we may conclude that either $\sqrt{ } Q$ is linear in $\lambda$ or that the left side of (2.21) is identically equal to zero. Thus,

either

(i) $\quad B^{2}-4 A C \equiv 0$,

or

and

$$
\text { (ii) } T Q_{\omega}-T^{\prime} Q \mp \frac{k T}{\omega} Q_{1} \equiv 0,
$$

$$
\pm \omega^{2} T Q_{1} Q_{\omega} \mp 2 \omega Q\left[\omega T^{\prime} Q_{1}-2 T Q_{1}-\omega T Q_{1, \omega}\right]-\frac{4 \lambda^{2} k T Q}{\omega^{3}}-\omega k T Q_{1}^{2} \equiv 0 .
$$

However, if $B^{2}-4 A C \equiv 0$, then in order that $\sqrt{ } Q$ may be real, $A$ must be nonnegative. But the last equation in (2.17) then implies that both $A$ and $k^{\prime}$ must vanish. From the expression for $A$ in (2.16) we conclude that $k \equiv 0$ and we excluded this case by our assumptions at the beginning of this section. Thus, under the assumptions already made, Eq. (2.14) is an identity if and only if (2.22) and (2.23) are satisfied simultaneously. Here two cases may be distinguished by the choice of the sign in front of some of the terms. Accordingly, choosing the second sign in both the equations, we have from (2.22),

$$
T A^{\prime}-T^{\prime} A+\frac{k T}{\omega} A_{1}=0,
$$

or, replacing $A_{1}$ by means of $(2.20)$,

$$
2 T A^{\prime}-T^{\prime} A+\frac{2 k}{\omega}\left(\frac{\omega}{k}\right)^{\prime} T A=0,
$$


and similar expressions with $B$ and $C$ in place of $A$. These may be integrated to yield

$$
A=a_{1} \frac{k}{\omega} \sqrt{ } T, B=b_{1} \frac{k}{\omega} \sqrt{ } T, C=c_{1} \frac{k}{\omega} \sqrt{ } T,
$$

where $a_{1}, b_{1}$ and $c_{1}$ are constants, not all zero.

Assuming that $a_{1} \neq 0$ so that $A \neq 0$, we may write $B$ and $C$ in terms of $A$ and, using (2.20), write $B_{1}$ and $C_{1}$ in terms of $A_{1}$ so that (2.23) will now become

$$
\omega\left(a_{1} \lambda^{2}+b_{1} \lambda+c_{1}\right)\left[2 A\left(\omega T^{\prime} A_{1}-2 T A_{1}-\omega T A_{1}^{\prime}\right)-\omega T A_{1} A^{\prime}-k T A_{1}^{2}\right]-\frac{4 a_{1} \lambda^{2} k T A}{\omega^{3}} \equiv 0 .
$$

If both $b_{1}$ and $c_{1}$ are zero, we revert back to the first case, $B^{2}-4 A C \equiv 0$. Thus, a necessary condition that the above may vanish identically is $T A \equiv 0$. But this means that $T \equiv 0$ and by $(2.20)$ this implies that $B^{2}-4 A C \equiv 0$.

We shall now choose the first sign in (2.22) and (2.23). Eq. (2.22) will vanish identically if and only if

$$
\frac{\omega}{k} T^{\prime}+2\left(\frac{\omega}{k}\right)^{\prime} T=0
$$

which may be integrated to yield

$$
T=d k^{2} / \omega^{2}, \quad d=\text { constant }
$$

By $(2.20)$, we have

$$
Q_{1}=2\left(\frac{\omega}{k}\right)^{\prime} Q+\frac{\omega}{k} Q_{\omega},
$$

which may be used to eliminate $Q_{\omega}$ from (2.23) to yield

$$
k \omega T Q_{1}\left(\frac{\omega}{k}\right)^{\prime}+\omega\left(\omega T^{\prime} Q_{1}-2 T Q_{1}-\omega T Q_{1, \omega}\right)+\frac{2 \lambda^{2} k T}{\omega^{3}} \equiv 0 .
$$

This is a quadratic in $\lambda$ and the coefficients must all vanish. Hence

$$
\begin{gathered}
k \omega T\left(\frac{\omega}{k}\right)^{\prime} A_{1}+\omega\left(\omega T^{\prime} A_{1}-2 T A_{1}-\omega T A_{1}^{\prime}\right)+\frac{2 k T}{\omega^{3}}=0, \\
k \omega T\left(\frac{\omega}{k}\right)^{\prime} B_{1}+\omega\left(\omega T^{\prime} B_{1}-2 T B_{1}-\omega T B_{1}^{\prime}\right)=0, \\
k \omega T\left(\frac{\omega}{k}\right)^{\prime} C_{1}+\omega\left(\omega T^{\prime} C_{1}-2 T C_{1}-\omega T C_{1}^{\prime}\right)=0 .
\end{gathered}
$$

The two equations of (2.27) may be integrated to yield

$$
B_{1}=b T / k \omega, \quad C_{1}=c T / k \omega,
$$

where $b \neq 0, c \neq 0$ are constants. The equation (2.26) may be rewritten as

$$
\left(A_{1} k \omega / T\right)^{\prime}=2 k^{2} / T \omega^{4} \text {. }
$$


Replacing $T$ by means of (2.25), this equation may be integrated to yield

$$
A_{1}=-\frac{2 k}{\omega^{4}}+a \frac{k}{\omega^{3}}, \quad a=\text { constant }
$$

Since $(\omega / k) A_{1}=\left(A \omega^{2} / k^{2}\right)^{\prime}$ by $(2.20)$ and similar expressions hold for $B_{1}$ and $C_{1}$, we may now calculate $A, B$ and $C$ from (2.28) and (2.29). Thus

$$
A=\frac{k^{2}}{\omega^{4}}\left(a_{1} \omega^{2}-a \omega+1\right) . \quad B=\frac{k^{2}}{\omega^{3}}\left(b_{1} \omega-b d\right), \quad C=\frac{k^{2}}{\omega^{3}}\left(c_{1} \omega-c d\right) .
$$

Thus in order that (2.14) may be an identity, the expressions on the right side of $(2.30)$ must equal the corresponding expressions in (2.16), giving us three differential equations for $k$ as a function of $\omega$. We shall show that these equations are incompatible. The first equation of (2.17) with $A, B$ and $C$ replaced by (2.30) will yield $k$ as a function of $\omega$ given by

$$
k^{2}=-\mu^{2} \omega^{3} /\left[a_{1} \omega^{3}+\left(b_{1}-a\right) \omega^{2}+\left(1-b d+c_{1}\right) \omega-c d\right] .
$$

We note that the coefficients of $\omega$ in the denominator of (2.31) cannot all vanish simultaneously. Further, the second equation of (2.17) yields

$$
\frac{2 k^{\prime}}{k}=\frac{b d-2}{\omega}-2 a_{1} \omega+\left(2 a-b_{1}\right) \text {. }
$$

This may be compared with the derivative of $k$ from $(2.31)$ and we find that in order that $\omega \neq$ constant, one of the following conditions must hold:

or

(i) $\quad a_{1}=0, \quad 2 a=b_{1} \neq 0, \quad b d=3, \quad c_{1}=2, \quad c=0$,

or

(ii) $a_{1}=0, \quad a=b_{1}=0, \quad b d=4, \quad c=0$,

(iii) $\quad a_{1}=0, \quad a=b_{1}=0, \quad b d=5, \quad c_{1}=4$.

Yet the expressions for $C$ given by (2.16) and (2.30) do not become equal in any of the above three cases. For example, in the last case, we have from (2.31) and (2.16),

$$
C=-\mu^{2}+\left(k^{2} / 4 \omega^{2}\right)
$$

but by $(2.30)$ and $(2.31)$

$$
C=-\mu^{2}+\left(4 k^{2} / \omega^{2}\right)
$$

Thus we conclude that (2.14) is not an identity and $\lambda$ may be solved as a function of $\omega$ so that by Lemma 2.1, we have the following theorem:

THEOREM 2.1. If the streamlines and isobars do not coincide in the steady twodimensional flow of an incompressible viscous fluid and if the vorticity remains constant along the isovels, then there exist constants $\gamma$ and $\lambda$ such that

$$
k=k(\gamma p+\lambda \psi) \text { and } \omega=\omega(\gamma p+\lambda \psi) .
$$

Here we have introduced the constant $\gamma$ to include the case $\omega=\omega(\psi)$. However, in what follows, we shall assume that $\gamma=1$ and $k=k(\tau), \omega=\omega(\tau)$ where $\tau=p+\lambda \psi$. 
The Gauss equation in (2.1) may now be written as

so that

$$
\frac{d}{d \tau}\left(\frac{\tilde{G}-\lambda \tilde{F}}{k \tilde{J}}\right)=0
$$

$$
\left(\lambda k_{p}+\omega\right) / k \omega_{p}=c, \quad c=\text { constant. }
$$

If $c=0$, then it follows (Eq. (1.5) of [3]) that $k=k(\alpha)$ and the flow will be a special case of the Prandtl-Meyer flow discussed in [3]. We note that the stream function is first deduced by Jeffery [6] and eliminate this case from further discussion and assume that $c \neq 0$.

We shall rewrite $(2.32)$ as

$$
\omega\left(k^{\prime}+\tau^{\prime}\right)=c k+(\omega-\lambda) k^{\prime}
$$

Similarly, the vorticity equation (2.6) may be written as

$$
\frac{\omega}{2 k} \frac{\tilde{E}}{\widetilde{J}}=\frac{1}{\mu}\left(k^{\prime \prime}+\tau^{\prime \prime}\right) \omega_{p},
$$

or

$$
\frac{\omega\left(k^{\prime}+\tau^{\prime}\right)}{k(\omega-\lambda)}=\frac{2\left(k^{\prime}+\tau^{\prime}\right)\left(k^{\prime \prime}+\tau^{\prime \prime}\right)}{\mu^{2}+\left(k^{\prime}+\tau^{\prime}\right)^{2}} .
$$

Using (2.33) to replace the numerator of the left side of (2.35), we may integrate this equation to yield

$$
k^{\prime}+\tau^{\prime}= \pm \sqrt{b(\omega-\lambda)^{c} k-\mu^{2}}, \quad b \neq 0, \quad \text { constant. }
$$

Using (2.33) again to replace $k^{\prime}+\tau^{\prime}$ in (2.36), we may integrate this equation to obtain $k$ as a function of $\omega$ :

$$
\begin{array}{rlrl} 
\pm \sqrt{b(\omega-\lambda)^{c} k-\mu^{2}} & =\frac{b}{2 c} \omega(\omega-\lambda)^{c}-\frac{b(\omega-\lambda)^{c+1}}{2 c(c+1)}+a & & \text { if } \quad c \neq-1 \\
& =\frac{b}{2} \ln |(\omega-\lambda)|-\frac{b}{2} \frac{\omega}{\omega-\lambda}+a \quad & \text { if } \quad c=-1 .
\end{array}
$$

where $a=$ constant.

These expressions may now be used to integrate equation (2.36) so that $\tau=p+\lambda \psi$ is given as a function of $\omega$ :

$$
\begin{aligned}
\tau= & \frac{b(\omega-\lambda)^{c+1}(c \omega+2 \lambda)}{2 c(c+1)(c+2)}-\frac{b(\omega-\lambda)^{c}(c \omega+\lambda)^{2}}{4 c^{2}(c+1)^{2}}-\frac{a^{2}+\mu^{2}}{b(\omega-\lambda)^{c}}+\frac{a c \omega}{c+1}+d \\
= & \left.-\frac{b \omega}{4(\omega-\lambda)}+\frac{b\left(4 \omega-\lambda^{2}\right)}{16(\omega-\lambda)^{2}}-\frac{b}{2} \ln |\omega-\lambda|-\frac{a^{2}+\mu^{2}}{b}(\omega-\lambda)^{2}+d \quad \text { or } \quad-2, \quad \text { if } \quad c=-2,38\right) \\
= & (b-a)(\omega-\lambda) \ln |\omega-\lambda|-\frac{b}{4}(\omega-\lambda)[\ln |\omega-\lambda|]^{2}+\left(a-b-\frac{a^{2}+\mu^{2}}{b}\right)(\omega-\lambda)+a \omega \\
& -\frac{b \omega^{2}}{4(\omega-\lambda)}+\mathrm{d} \quad \text { if } \quad c=-1 .
\end{aligned}
$$


In order to determine the flow in the physical plane, it is convenient to change the independent variables $p$ and $\psi$ in $x, y$ and $\alpha$ to the variables $\tau$ and $p$ so that $\psi=(\tau-p) / \lambda$ and

$$
\frac{\partial x(\tau, p)}{\partial p}=x_{p}-\frac{1}{\lambda} x_{\psi}, \frac{\partial x(\tau, p)}{\partial \tau}=\frac{1}{\lambda} x_{\psi},
$$

and similar expressions hold for $y$ and $\alpha$.

From (1.4), with $\phi$ replaced by the pressure $p$, we have

$$
\left.\alpha_{p}=\frac{1}{2 k}\left|\frac{\tilde{F}}{\widetilde{J}}+\mu \omega_{p}\right|, \quad \alpha_{\psi}=\frac{1}{2 k} \mid \frac{\tilde{G}}{\widetilde{J}}+\mu \omega_{\psi}\right],
$$

which, when simplified, become

$$
\alpha_{p}=\frac{\lambda \mu^{2} \omega+c^{2} k^{2}}{2 \lambda \mu k(\omega-\lambda)} \omega_{p}-\frac{c}{2 \lambda \mu}, \quad \alpha_{\psi}=\frac{\lambda \mu^{2} \omega+c^{2} k^{2}}{2 \mu k(\omega-\lambda)} \omega_{p}
$$

so that

$$
\frac{\partial \alpha}{\partial \tau}=\frac{\lambda \mu^{2} \omega+c^{2} k^{2}}{2 \lambda \mu k(\omega-\lambda)} \omega_{p}, \quad \frac{\partial \alpha}{\partial p}=-\frac{c}{2 \lambda \mu} .
$$

To facilitate the calculation of $x$ and $y$ we write these formulas in yet another form using the vorticity equation (2.34), which may be written as

$$
\frac{d}{d \tau}\left[\frac{k^{\prime}+\tau^{\prime}}{\sqrt{ } k}\right]=\frac{\mu^{2} \omega \omega_{p}+c k\left(k_{p}+1\right)}{2 k^{3 / 2}(\omega-\lambda)}=\frac{\lambda \mu^{2} \omega+c^{2} k^{2}}{2 \lambda k^{3 / 2}(\omega-\lambda)}-\frac{c}{2 \lambda \sqrt{ } k} \quad \text { by (2.32). }
$$

Thus (2.39) now may be written as

From (1.3),

$$
\frac{\partial \alpha}{\partial \tau}=\sqrt{ } k \frac{d}{d \tau}\left(\frac{k^{\prime}+\tau^{\prime}}{\mu \sqrt{ } k}\right)+\frac{c}{2 \lambda \mu} \text { and } \quad \frac{\partial \alpha}{\partial p}=-\frac{c}{2 \lambda \mu} .
$$

$$
\begin{aligned}
& \frac{\partial x}{\partial \tau}=\frac{1}{\lambda \sqrt{2 \rho k}}\left(\frac{\tilde{F}}{\tilde{J}} \cos \alpha-\sin \alpha\right), \\
& \frac{\partial x}{\partial p}=\frac{1}{\lambda \sqrt{2 \rho k}}\left(-\frac{\left(k^{\prime}+\tau^{\prime}\right)}{\mu} \cos \alpha+\sin \alpha\right) .
\end{aligned}
$$

The second equation may be integrated and compared with the first by means of (2.40). Similar calculations may be made for $y$, so that we have

$$
z=z_{0}+\frac{2}{c \sqrt{2 \rho k}}\left[\mu-i\left(k^{\prime}+\tau^{\prime}\right)\right] e^{i x}, \quad z_{0}=x_{0}+i y_{0}=\text { constant. }
$$

If $(r, \theta)$ are the polar coordinates of the point $(x, y)$, then this expression together with (2.36) yields

$$
(\omega-\lambda)^{c}=\frac{c^{2} \rho r^{2}}{2 b}
$$

and by (2.40),

$$
\theta=\frac{c^{2}}{2 \lambda \mu} \int \frac{k}{\omega-\lambda} d \omega-\frac{c}{2 \lambda \mu} p
$$


The relation (2.41) shows that $\omega=\omega(r)$ so that the flows will be Jeffery flows [6].

The integral in the right side of (2.42) may be evaluated using (2.37). $\omega$ and $p$ may be eliminated from the resulting expression using (2.41) and (2.38) and an expression for the stream function $\psi$ in polar coordinates is thus obtained as

$$
\begin{aligned}
\psi & =\frac{\lambda \mu}{c} \theta-\frac{\lambda \rho}{4(c+1)} r^{2}+A \cdot r^{2+(2 / c)}+B \cdot \ln r+\psi_{0} \quad \text { if } \quad c \neq-1 \quad \text { or }-2, \\
& =-\mu \theta+A r+\frac{\rho(1-\lambda)}{2} r^{2}+B \ln r+\psi_{0} \quad \text { if } \quad c=-2, \\
& =-2 \mu \theta-\frac{\lambda \rho}{4} r^{2}+A \ln r+B(\ln r)^{2}+\psi_{0} \quad \text { if } \quad c=-1,
\end{aligned}
$$

where $A, B$ and $\psi_{0}$ are constants.

We conclude this section with the following theorem:

THEOREM 2.2. If the streamlines do not coincide with the isobars in the steady twodimensional flow of a viscous incompressible fluid, and if the vorticity is constant on the isovels, then the isovels are either (i) concentric circles and the flow is a Jeffery flow, or (ii) straight lines and the flow is a plane Couette flow.

The second case arises out of a special case of $\omega=\omega(\psi)$ discussed in [3].

3. Streamlines and isobars coincide. In this section, along with our assumption $k=k(\omega)$, we also assume that $p=p(\psi)$ so that the streamlines and isobars coincide. We now use the system (1.1) with the curvilinear coordinates $\phi=$ constant taken as orthogonal to the streamlines $\psi=$ constant so that $\widetilde{F} \equiv 0$. As in the last section, we assume that $\omega_{\phi} \neq 0$. Thus we now have an overdetermined system of five equations for three unknowns $\omega, k$ and $p$ given by:

$$
\begin{gathered}
-\left(\frac{\tilde{E}}{\tilde{J}}\right)_{\psi}=\frac{\omega}{2 k} \frac{\tilde{E}}{\widetilde{J}} \quad \text { (vorticity), } \\
\left(\frac{\tilde{E}}{\tilde{J}} \frac{p_{\psi}}{k}\right)_{\psi}=0 \quad \text { (Gauss), } \\
k_{\phi} \omega_{\psi}-k_{\psi} \omega_{\phi}=0, \\
p_{\phi} \equiv 0, \\
\left.\omega_{\psi}\left(\mu^{2}+k^{\prime 2}\right)+k^{\prime}\left(p_{\psi}+\omega\right)=0 \quad \text { (orthogonality, } \tilde{F} \equiv 0\right),
\end{gathered}
$$

where, ${ }^{\prime}=d / d \omega, \tilde{E}=\omega_{\phi}^{2}\left(\mu^{2}+k^{\prime 2}\right)$ and $\tilde{J}=\mu \omega_{\phi}\left(p_{\psi}+\omega\right)$.

We note here that if $\widetilde{J} \equiv 0$, then it may be easily proved along the lines given in [3] that the vorticity must remain constant. In what follows, we will assume that vorticity is not a constant in the flow.

To find functions $\omega(\phi, \psi), k(\phi, \psi)$ and $p(\psi)$ which satisfy the above five equations, we shall apply a series of integrability conditions as in the last section. From the Gauss equation (3.2) and the orthogonality condition (3.5), we calculate $\omega_{\phi}$ and $\omega_{\psi}$ and apply the integrability condition $\omega_{\phi \psi}=\omega_{\psi \phi}$ to obtain an expression for $\omega$ involving $k, p$ and their derivatives. This, together with (3.1) and (3.4), will enable us to conclude that the pressure must remain constant everywhere. 
Thus, if $p$ ₹ constant, the Gauss equation (3.2) yields

$$
\omega_{\phi}=\frac{\mu k(\dot{p}+\omega)}{\dot{p}\left(\mu^{2}+k^{\prime 2}\right)} f(\phi),
$$

where $=d / d \psi$ and $f$ is an arbitrary function of $\phi$. Further, by (3.5),

$$
\omega_{\psi}=-\frac{k^{\prime}(\dot{p}+\omega)}{\mu^{2}+k^{\prime 2}} .
$$

The integrability condition $\omega_{\phi \psi}=\omega_{\psi \phi}$ yields

$$
\left(k k^{\prime \prime}-k^{\prime 2}\right) \dot{p}(\dot{p}+\omega)^{2}-\omega k\left(\mu^{2}+k^{\prime 2}\right) \ddot{p}=0 .
$$

On the other hand, when (3.6) and (3.7) are used to eliminate the derivatives of $\omega$, the vorticity equation $(3.1)$ reduces to

$$
2 k^{\prime 2} \dot{p}(\dot{p}+\omega)^{2}+2 k\left(\mu^{2}+k^{\prime 2}\right) \ddot{p}-\omega\left(\mu^{2}+k^{\prime 2}\right) \dot{p}=0 .
$$

Eliminating $\ddot{p}$ from (3.8) and (3.9), we have, since $\dot{p} \neq 0$,

$$
2\left(k k^{\prime \prime}-k^{\prime 2}\right)(\dot{p}+\omega)^{2}+2 \omega k^{\prime 2}(\dot{p}+\omega)-\omega^{2}\left(\mu^{2}+k^{\prime 2}\right)=0 .
$$

This is a quadratic for $\dot{p}+\omega$ whose coefficients are functions of $\omega$ and clearly it is not an identity. Thus we may conclude that $\dot{p}+\omega$ must be a function of $\omega$. Since $p=p(\psi)$ and $\omega \neq \omega(\psi)$, this would only mean that $\dot{p}=$ constant so that by (3.8) we have

which may be integrated to yield

$$
k k^{\prime \prime}-k^{\prime 2}=0 \text {, }
$$

$$
k=k_{0} e^{a \omega} ; \quad k_{0}, a=\text { constant. }
$$

However, when this value of $k$ is used in (3.9), we get

$$
a^{2} k_{0}^{2}(\dot{p}+\omega) e^{2 a(\omega)}-\mu^{2} \omega=0,
$$

which implies that $\omega \equiv$ constant.

Thus we have the following theorem:

THEOREM 3.1. If the vorticity remains constant along the isovels in the steady twodimensional flow of an incompressible viscous fluid and if the streamlines and isobars coincide, then either (i) the vorticity is constant everywhere or (ii) the pressure is constant everywhere.

If the pressure is constant everywhere, then the Gauss equation (3.2) becomes redundant and (3.6) is no longer valid. However, the vorticity equation (3.1) may be simplified as

$$
\left.\left(\mu^{2}+k^{\prime 2}\right) \omega_{\phi \psi}+\left(2 k^{\prime} k^{\prime \prime}-\mu^{2}-k^{\prime 2}\right) \omega_{\phi} \omega_{\psi}+\mid k^{\prime}+\frac{\omega}{2 k}\left(\mu^{2}+k^{\prime 2}\right)\right] \omega_{\phi}=0 .
$$

When $\omega_{\psi}$ and $\omega_{\phi \psi}$ are eliminated from this equation using (3.7), we get

which may be integrated to yield

$$
\mu^{2}+k^{\prime 2}=2 k k^{\prime \prime}
$$

$$
k=\frac{c^{2}}{4}(\omega+b)^{2}+\mu^{2} / c^{2} ; \quad b, c=\text { constant }, \quad c \neq 0 .
$$


Using (3.10) to replace $k$ from (3.5) and integrating, we have $\omega$ as a function of $\phi$ and $\psi$ given implicitly as

$$
\begin{aligned}
& \omega+\frac{b}{2} \ln |\omega|+\frac{4 \mu^{2}}{b c^{4}} \ln \left|\frac{\omega}{\omega+b}\right|+\frac{2}{c^{2}} \psi=\Phi \quad \text { if } \quad b \neq 0 \\
& \frac{c^{2}}{2} \omega-\frac{2 \mu^{2}}{c^{2} \omega}+\psi=\Phi \quad \text { if } \quad b=0 .
\end{aligned}
$$

where $\Phi$ is an arbitrary function of $\phi$.

To obtain the flow in the physical plane, we have, from (1.4),

$$
\alpha=\int \frac{d \omega}{2 k},
$$

so that by $(3.10)$

$$
\alpha-\alpha_{0}=\arctan \left[\frac{c^{2}}{2 \mu}(\omega+b)\right\rfloor, \quad \alpha_{0}=\text { constant } .
$$

By means of (3.5), (3.10) and (3.12), we may now calculate $z=x+i y$ from (1.3):

$$
z=z_{0}+\frac{c^{i x_{0}}}{2 \mu \sqrt{2 \rho}}\left[2 \mu \ln |\omega|+i\left\{2 \psi+c^{2}(\omega+b \ln |\omega|)\right\}\right], z_{0}=x_{0}+i y_{0}=\text { constant }
$$

From this we have $\omega$ as a function of $x$ and $y$ as

$$
\omega=\exp \left|\frac{\sqrt{2 \rho}}{c}\left[A\left(x-x_{0}\right)+B\left(y-y_{0}\right)\right]\right|, A^{2}+B^{2}=1,
$$

and the streamfunction

$$
\psi=\frac{\mu \sqrt{2 \rho}}{c}\left[A_{1}\left(x-x_{0}\right)+B_{1}\left(y-y_{0}\right)\right]-\frac{c^{2}}{2} \exp \left|\frac{\sqrt{2 \rho}}{c}\left[A\left(x-x_{0}\right)+B\left(y-y_{0}\right)\right]\right|,
$$

where

$$
A_{1}=-B-\frac{b c^{2}}{2 \mu} A \quad \text { and } \quad B_{1}=A-\frac{b c^{2}}{2 \mu} B
$$

\section{REFERENCES}

[1] M. H. Martin, The flow of a viscous fluid I, Arch. Rat. Mech. Anal. 41, 266-286 (1971)

[2] M. H. Martin, Plane rotational Prandtl-Meyer flows, J. Math. and Phys. XXIX, 76-89 (1950)

[3] K. Govindaraju, The flow of a viscous fluid II, Arch. Rat. Mech. Anal. 46, 66-80 (1972)

[4] C. Riquier, Les systèmes d'équations aux derivées partielles, Paris (1910)

[5] J. M. Thomas, Riquier's existence theorems, Ann. Math., 30, 285-310 (1929)

[6] G. B. Jeffery, The two-dimensional steady motion of a viscous fluid, Phil. Mag. 28, 455-458 (1915)

[7] R. Berker, Integration des équations de mouvement d'un fluide visqueux incompressible, Stromungs Mechanik II, Handbuch der Physik, VIII/2, 40-41 (1963) 